\title{
Use of Image Processing to Easy e-content Reader for Physically Differently-Abled Personnel
}

\author{
A. H. R. P. Mithrasena ${ }^{1}$, D. M. R. Kulasekara ${ }^{2}$ and N. Wedasinghe $^{3}$ \\ 1, 2,3 Department of Information Technology, Faculty of Computing, \\ General Sir John Kotelawala, Defence University, Sri Lanka \\ ${ }^{1}$ ruwani_mithrasena@yahoo.com, ${ }^{2}$ dmrangak@gmail.com, ${ }^{3}$ niroshaw@ hotmail.com
}

\begin{abstract}
Differently-abled or disability is distinct as limitation of a person's ability to carry out the activities of daily living, to the level that he or she may need help in doing so. The differentlyabled personnel, especially people with various amputations face the issue of not being able to use the devices that allow them to access e-content properly. In this paper, here suggest an application that is able to scroll the page of an e-content with the help of human face. The face is identified and tracked in real-time to use their actions for scrolling events. The basic strategy for detection is fast extraction of the face of the image in front of the screen with image processing technology. Between-the-Eyes is selected as a face representative because of its features are common to most people and is easily seen for a varied range of face orientation. After identifying the face, the head movement is used to trigger the scrolling event based on the suggested proposed system
\end{abstract}

Keywords: E-content, Image Processing, Amputations, Trigger

\section{Introduction}

The World Health Organization estimates that about 650 million (about 10 or 15 percent of the world population) around the world are differently abled people and of this its estimate, 80 percent live in developing countries. According to Department of Census and Statistics Sri Lanka 2012, the number of people disadvantaged due to disability stands at $1,617,924$. This was updated each 10 years of time within this time period this can be changed due to natural disasters occur. Due to the 'civil war' that ravaged the country, during the last few years had affected many mentally and physically. Number of people, both civilians and fighters, on both sides have been affected physically and psychologically. Many have lost their limbs and hands due to artillery fire and due to bombing from the air and many have become mentally 
deranged having witnessed the loss of their kith and kin. In the present world the global access to knowledge has been ensured with the rise of technology. However, this is not the case when it comes to differently-abled personnel. Regardless of the technological advancements even now the access to e-content is still limited to these personnel and facilitating them accordingly still remains as an active area of research for computer scientists. This solution that's going to propose as explained below will act as a platform to bridge the gap between the e-content and the differently-abled personnel.

The main objective of this study is to overcome the main problems faced by the disability people having with movements of hands when reading document using scroll down the page. The hypothesis identified is by using head movements recognition or face recognition method difficulty of scroll down pages issues could be solved

The problem as identified is that the differently-abled personnel, especially people with various amputations face the issue of not being able to use the devices that allow them to access e-content properly (Bulling, et al., 2011). This is due to the fact that almost all these devices use input devices such as keyboards, mice or touch screens. In order to grasp the essence of e-content which comes in the forms of digital textbooks, articles, videos or multimedia, the differently-abled personnel requires the use of special hardware or the use of prosthetic limbs which are both very expensive to join. Even if voice commands address this issue to some extent, it hasn't been able to provide a complete solution to the problem (Sindhu et al., 2014). The main objective of this development is to focuses on using image processing technology to effectively use the face of the user of the proposed platform as a fully functional input device and thereby reducing the need of a user to interact with a mouse or a keyboard during their learning experience. In order to do this, face detection must be done by the proposed platform. The rest of the sections will describe the design, how the system works and conclusion. 


\section{Literature Review}

In recent years, a lot of research has been done in the area of face recognition using image proceeding technology and there were different projects carrying out by universities regarding image processing for identifying objects or facial movements etc. A growing number of researchers use video-based eye tracking to study eye movements in natural environments. These types of researches had involved in projects like automated driving vehicle (Jain et al., 2014) and to identify eye movements in reading (GIDJRIDJT, 2014). This has led to important advances in our understanding of how the brain processes, tasks, and of the role that the visual system plays in this. Eye movement analysis has a long history as a tool to investigate visual behaviour.

In the past few years, face recognition using image processing technology has received a significant attention and regarded as one of the most positive applications in the field of image analysis (W. Zhao, et al,2000) The human faces represent complex, multidimensional, meaningful visual stimulating. Developing a computational model for face recognition is difficult (Turk M \& Pentland A, 1991). Face detection can be considered as fundamental part of face recognition systems according to its ability to focus computational resources on the part of an image having a face.

The process of face detection in images is difficult because of inconsistency present across human faces such as: pose; expression; position and orientation; skin colour; existence of glasses or facial hair; changes in camera gain; lighting conditions; and image resolution (Phil Brimblecombe, 2002). At the same time, improvements in many fields such as: face detection (P. Viola \& M.J. Jones, 2001); tracking (Minyoung Kim, et.al, 2008); and recognition; pattern recognition; and image processing contributed meaningfully to research in automatic facial expression recognition

At present, a lot of research has been done in the area of helping differently-abled people. All the work has a general aim: to help differently-abled people to make their day to day activities easily. There are very few applications design in this context like hands-free PC control" controlling of mouse cursor using eye movement (Gupta and 
Rathi, 2012) smart scroll application for smart phones and tablets. But still there is no desktop application for scroll page of a document. There are several applications which have been developed using face recognition using image processing technology like automated driving vehicle using image processing and also there are several systems which have been developed to help physically differently abled people like eyeball motion controlled wheelchair using IR sensors (Jain et al., 2015), gesture controlled home automation and different vehicles which can used by the user themselves.

The main faults which can talk about these systems were the systems with hardware integration may be very hard to use for a differently abled people. And also some of these systems may cost huge amount which cannot be afforded to them. Sometimes these systems may be too complex for them to handle due to the lack of technical knowledge they have. So due to some of the mentioned reasons these systems were failing to target the audience. The proposed system is cost effective as well as easy to use by, any person because its' simple and affecting system for the current problem.

Face detection should be performed before recognition system. This is done to extract relevant information for face and facial expression analysis. Two classes of techniques for face representation and relevant information extraction. And geometrical feature extraction relies on parameters of distinctive features such as eyes, mouth and nose. At the same time, a face is represented as an array of pixel intensity values suitably pre-processed in appearance based approaches (texture). This array is then compared with a face template using a suitable metric. Research compared the performances of these representation techniques in face recognition.

Therefore, according to the complexity of face detection process, many applications based on human face detection have been developed recently such as surveillance systems, digital monitoring, intelligent robots, notebook, PC cameras, and digital cameras and $3 \mathrm{G}$ cell phones. These applications play an important role in our life. Nevertheless, the algorithms of the applications are complicated and hard to meet realtime requirements of specific frame-rate. Over the past decade, many approaches for 
improving the performance of face detection have been proposed. At the same time, many literature studies focused on survey on face detection techniques

Face recognition is a biometric method that employs computerised methods to verify or recognize the identity of a living person based on user's physical characteristics. In general, a biometric identification system makes use of either physical characteristics (such as a fingerprint, iris pattern, or face) or behaviour patterns (such as hand-writing, voice, or key-stroke pattern) to recognise a person. Because of human inherent protectiveness of the user's eyes, some people are hesitant to use eye identification systems. Face recognition has the benefit of being a passive, non-intrusive system to prove personal identity in a "natural" and friendly way. Based on these results suggesting to develop a smart scroll applications for the desktop using image processing technology.

\section{Methodology}

Description of the top level diagram as capturing the face of the user from the web camera of the device such as laptop, desktop or tablet etc. It acts as the main input to the system. Through image processing, the facial features such as the eyes and the absolute distance between the eyes and nose should be detected (Sawettanusorn, et al., 2004). Other nodal point such as the width of the nose, depth of the eye sockets, the length of the jaw line, the relative distance between the eyes and nose should also be detected in order to properly function the proposed platform. Then Extract the exact feature which is needed to develop the system. Then track the face movements like 
how the head nods when reading. Based on that the scroll bar of the reading material can be control.

\section{Figure 01: Top Level Design of the Proposed System}

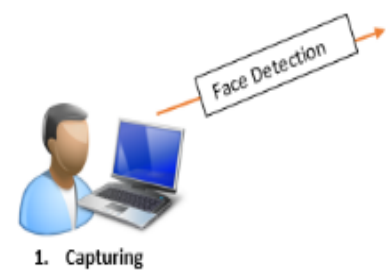

The foremost requirement is to capture the image and that can be done by using cameras
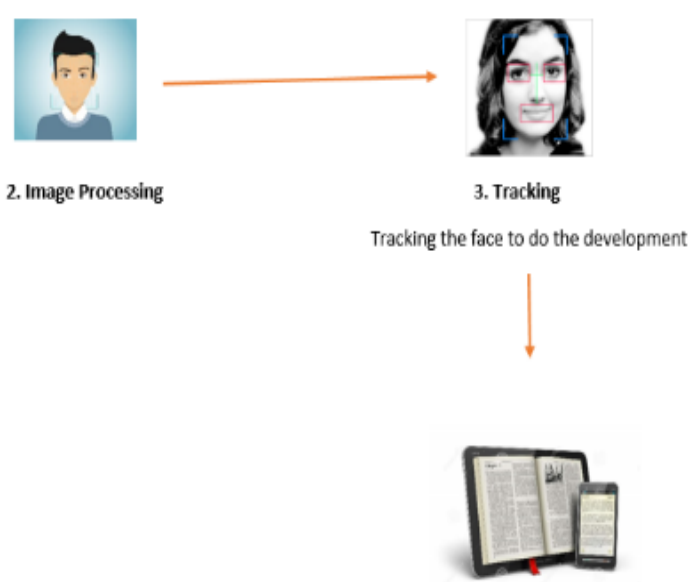

4. Developing the software solution

Declaring the necessary nodal points of the face developing the solution

The solution as suggested by this section focuses on using image processing technology to effectively use the face of the user of the proposed platform as a fully functional input device and thereby reducing the need of a user to interact with a mouse or a keyboard during their learning experience. In order to do this, face detection must be done by the proposed platform. Through image processing, the facial features such as the eyes and the absolute distance between the eyes and nose should be detected. Other nodal point such as the width of the nose, depth of the eye sockets, the length of the jaw line, the relative distance between eyes and nose should also be detected in order to properly function the proposed platform. Once this is done, the system could be calibrated to suit a person's face so that he could effectively use the proposed system.

The detected nodal points are then used to detect movements of the face such as nodding, moving the head up and down, moving the head sideways and even shoulder 
movement. By uniquely identifying these motions, each of them could be configured to achieve basic functionality like scrolling, clicking, accepting or rejecting etc.

Moreover by nodding the head sideways user will be able to select the required document that he or she want. That selected document will load to the PDF container. With the calibrate button user is able to calibrate his face. From that the machine can accurately detect the face.

As for an example, the system could be used by a differently-abled person to open and navigate through an e-book with the use of a series of facial motions such as by moving the head up or down to scroll up or down the page, by tilting the head sideways to go to the previous or the next page etc. The system also provides functionality to set the function associated with a facial movement accordingly to the need of the user which provides them the ability to personalise the experience. Further the system detects user's fatigue by the blink rate of the eyes and could be then effectively used to change the learning experience accordingly to keep the user focused. For an example, if the user is feeling tired after reading an e-book, he could then be switched to a song or any other interactive experience.

Since the system only hardware requirement is a webcam in order to fully utilize its functionality, this solution would prove to be a much more cost effective and efficient than which are currently available in the market.

\section{Conclusion}

This paper motivated on the analysis of the development of e-content reader using human eyes, application in all aspects. Originally, the problem domain was recognised and present commercial products that fall in a similar area were compared and contrasted by assessing their features and deficiencies. The usability of the system is very high, especially for its use with desktop applications. It shows accuracy and speed, which are necessary for many real time applications and which allow many differently abled users to enjoy many compute activities. In fact, it was possible to completely simulate a mouse without the use of the hands. 


\section{Scope for Future Research}

For further development the system detects user fatigue by the blink rate of the eyes and could be then effectively used to change the learning experience accordingly to keep the user focused. For an example, if the user is feeling tired after reading an ebook, he could then be switched to a song or any other interactive experience. Since the system only hardware requirement is a webcam in order to fully utilize its functionality, this solution would prove to be a much more cost effective and efficient than which are currently available in the market.

\section{Acknowledgment}

I would like to express my special thanks of gratitude to project supervisors and all the lectures of faculty of computing for their guidance and encouragement in carrying out this research project. I wish to express my gratitude to my parents and all my friends who helped me to do this successfully. 


\section{References}

Bulling, Ward, J, A. Gellersen, H \& Tröster, G. (2011) Eye movement analysis for activity recognition using electrooculography. Pattern Anal Mach Intell IEEE Trans, 33(4), 741-753.

Rathi.A \& Gupta, A.K.H.I.L. (2012). "HANDS-FREE PC CONTROL" CONTROLLING OF MOUSE CURSOR USING EYE MOVEMENT", International Journal of Scientific and Research Publications, 2(4), 5.

Savadi. C \& patil, V. (2014). Face Based Automatic Human Emotion Recognition. Int J Comput Sci Netw Secur IJCSNS, 14(7), 79.

Gupta, S, dholakiya , D \& barve, , S. (2012). "Real-Time Feature based Face Detection and Tracking I-Cursor. ", International Journal of Computer Applications, 48(2), 24.

Jain, H. Reddy, and S. Dubey, (2014) "Automated Driving Vehicle Using Image Processing,"

Jain, M., Puri, S., Unishree, S., (2015) Eyeball Motion Controlled Wheelchair Using IR Sensors. World Acad. Sci. Eng. Technol. Int. J. Comput. Electr. Autom. Control Inf. Eng. 9, 906-909.

M. Nehete, M. Lokhande and K. Ahire, (2013)"Design an Eye Tracking Mouse", International Journal of Advanced Research in Computer and Communication Engineering, 2(2)

O. Sawettanusorn, Y. Senda, S. Kawato, N. Tetsutani and H. Yamauchi,( 2004) "Detection of Face Representative Using Newly Proposed Filter", Journal of Signal Processing, 8(2),137-145.

U. L. Sindhu, A. Asha, S. Suganya, and M. Vinodha, (2014), "Face Recognition in Online Using Image Processing," Karpagam Inst. Technol. Int. J. Commun. Comput. Technol, 2 (13), 230-270. 